\title{
Maternally inherited hearing impairment in a family with the mitochondrial DNA A7445G mutation
}

\author{
Tim P Hutchin ${ }^{1}$, Nicholas J Lench ${ }^{1}$, Svetlana Arbuzova ${ }^{2}$, Alexander F Markham ${ }^{1}$ and \\ Robert F Mueller ${ }^{3}$
}

\author{
${ }^{1}$ M olecular M edicine Unit, St James's University Hospital, Leeds, UK; ${ }^{2}$ Interregional Medico-Genetic Centre, Donetsk, \\ Ukraine; ${ }^{3}$ Department of Clinical Genetics, St James's Hospital, Leeds, UK
}

\begin{abstract}
Despite the increasing number of reports of families with hearing impairment and mitochondrial DNA (mtDNA) mutations, the frequency of these mutations as causes of non-syndromic sensorineural hearing impairment (NSSHI) remains unknown. Mutations such as A1555G, A7445G and 7472insC have been found in several unrelated families implying they are more frequent than initially thought. We describe a family with NSSHI due to the presence of the homoplasmic mtDNA A7445G mutation in the tRNASer ${ }^{\text {(UCN) }}$ gene. This is the fourth such family described with this mutation, all of different genetic backgrounds. Our study also demonstrates the difficulties sometimes encountered in establishing mitochondrial inheritance of hearing impairment in some families. European Journal of Human Genetics (2001) 9, 56-58.
\end{abstract}

Keywords: hearing impairment; maternal inheritance; mitochondrial DNA mutation; A7445G; tRNASer(UCN)

Hearing impairment is the most common sensory defect in humans, affecting about 1 in 1000 live births and a similar number in childhood. At least half of these cases are due to genetic defects, including mutations of the mitochondrial DNA (mtDNA). Several mtDNA point mutations have been reported in families with maternally inherited NSSHI; the A1555G mutation in the 12S rRNA gene, which also causes hypersensitivity to aminoglycosides ${ }^{1,2}$ and four mutations affecting the tRN ASer ${ }^{\text {(UCN) }}$ gene - A7445G, 7472insC, T7510C and $T 7511 C .^{3-7}$ The frequency of these mutations in the general population has yet to be determined. Both the A1555G and A7445G mutations have been reported in different ethnic populations raising the possibility that such mutations are more common causes of hearing loss than initially thought.

Screening for mtDNA mutation is thus likely to be worthwhile in many cases of familial hearing impairment. Maternal inheritance can be difficult to confirm in smaller families or where few family members are affected, such as may be the case for heteroplasmic mutations. The presence of a phenocopy (ie hearing loss due to environmental factors)

Correspondence: Dr TP Hutchin, Molecular Medicine Unit, Clinical Sciences Building, St James's University Hospital, Beckett Street, Leeds, LS9 7TF, UK. Tel: +44113 206 5674; Fax: +44 113244 4475;

E-mail: t.p.hutchin@leeds.ac.uk

Received 3 July 2000; revised 14 August 2000; accepted

15 September 2000 may also confuse the inheritance pattern. Determining the mode of inheritance in familial cases is important in order to provide suitable DNA analysis as well as appropriate genetic counselling, most notably of the risk of passing on the hearing impairment.

We describe a family with non-syndromic hearing impairment affecting three generations (Figure1). Although the presence of both male and female affecteds and apparent transmission by both sexes initially suggested that hearing impairment in this family was transmitted in an autosomal dominant manner, subsequent analysis revealed a mutation in the mtDNA - A7445G - as the genetic cause of hearing impairment in the family.

The proband, IV:1, presented with difficulty in hearing in the classroom and was found to have an asymmetric bilateral sensorineural hearing impairment of $25 \mathrm{~dB}$ on the right and $50 \mathrm{db}$ on the left (average of $500 \mathrm{~Hz}, 1,2$ and $4 \mathrm{kHz}$ ). Her hearing impairment progressed so that by 20 years of age her loss on the right was $75 \mathrm{~dB}$ and $45 \mathrm{~dB}$ on the left. Her sister, $\mathrm{IV}: 2$, presented at 6 years of age with a mild symmetric bilateral sensorineural hearing impairment of $35 \mathrm{~dB}$. Again her hearing impairment progressed so that by 15 years of age she had a bilateral loss of $57 \mathrm{~dB}$. Their mother, III:2, had had a long-standing hearing loss. Assessment at the age of 28 years showed her to have a bilateral assymmetric sensorineural hearing loss of $50 \mathrm{~dB}$ on the right and $65 \mathrm{~dB}$ on the 
I

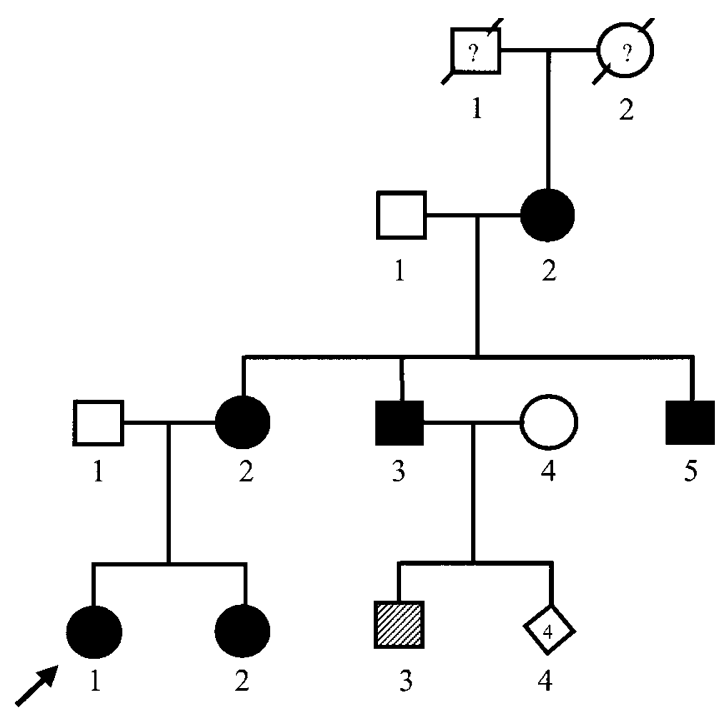

III

II

Figure 1 Pedigree of the family with non-syndromic hearing impairment. Solid symbols denote those with non-syndromic hearing impairment. Hearing loss in individual IV:3 (hatched symbol) was caused by glue ear. The proband is indicated by an arrow.

left. Her brother, III:3, had a bilateral symmetric sensorineural hearing loss of $67 \mathrm{~dB}$ on the left and $69 \mathrm{~dB}$ on the left, while her other brother III:5 had a bilateral symmetric sensorineural hearing impairment of $64 \mathrm{~dB}$ on the left and $66 \mathrm{~dB}$ on the right. Although initial enquiry suggested that one son of III:3, IV:3, also had sensorineural hearing impairment, further enquiry revealed this to be conductive secondary to middle ear disease.
To date five point mutations of the mtDNA have been reported in families with non-syndromic maternally inherited hearing impairment; the A1555G mutation in the $12 \mathrm{~S}$ rRNA gene, which also causes hypersensitivity to aminoglycosides $^{1,2}$ and four mutations affecting the tRNA $A^{\text {Ser(UCN) }}$ gene, A7445G, 7472insC, T7510C and T7511C. ${ }^{3-6}$

DNA was extracted from whole blood taken from four affected family members (III:2, III:3, IV:1 and IV:2) and an unaffected daughter of $1 \mathrm{II}: 3$, following informed consent. Analysis by PCR and restriction enzyme digestion showed the family did not carry the A1555G mutation but lacked the $X$ bal site at position 7440 , indicative of the A7445G mutation, which was homoplasmic in all four affected family members tested (Figure2). The presence of the A7445G mutation was confirmed by direct sequencing of the PCR product which also showed the absence of other mutations in the tRNASer ${ }^{(\mathrm{UCN})}$ gene associated with NSSHI. The mutation was not present in the daughter of the proband's uncle (IV:4).

The finding of the $A 7445 G$ mutation, which was not present in 176Caucasian controls screened, including 16 from the Ukraine (where the family originates), nor in 640 from previous studies, ${ }^{3}$ brings to four the number of families reported with hearing impairment and the A7445G mutation. ${ }^{3,7,8}$ mtDNA haplotype analysis of the three previous families with the A7445G mutation had shown they were unrelated. Similar analysis showed the family described here lacked the T4216C and G13708A base changes found in the New Zealand family ${ }^{8}$ and the $\mathrm{G} 14368 \mathrm{C}$ polymorphism of the Scottish family. ${ }^{9}$ No further sequence data was available on the Japanese family but since it is unlikely they are related to this family from Eastern Europe, the A7445G mutation has

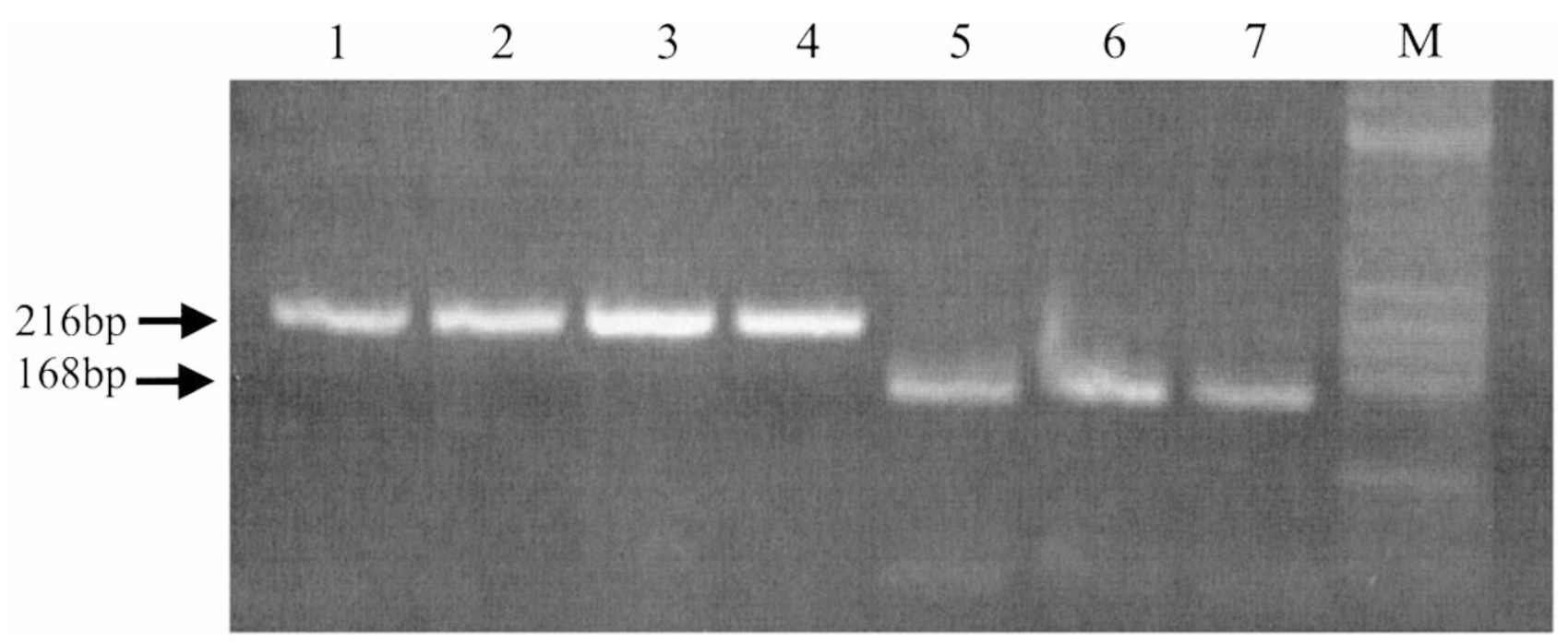

Figure 2 PCR-RFLP detection of the A7445G mutation. A $216 \mathrm{bp}$ fragment between nucleotide pairs 7392 and 7608 was amplified by PCR and digested with Xbal restriction enzyme. The normal product is cut into two fragments of 168 and 48 base pairs whereas the A7445G mutation abolishes this site. Affected individuals (IV:1, IV:2, III:2, III:3) are shown in lanes 1-4 and lack the Xbal site at position 7445. An unaffected daughter (IV:4) of the probands uncle (III:3) and two hearing controls are shown in lanes5-7. M = DNA marker pBR322 Haell digest. 
presumably arisen on four independent genetic backgrounds.

The presence of the A7445G mutation in four independent families provides further support that the mutation is pathogenic by itself though the reason for variation in penetrance between the families with the mutation is not known. Tissue specific differences in levels of heteroplasmy might explain such variation as may the presence of other, secondary, mtDNA mutations or nuclear DNA variation.

Unlike the other three families, that reported here showed no signs of palmoplantar keratoderma. The tissue specificity of mtDNA mutations causing hearing impairment, such as A7445G, is not fully understood but possible interactions with environmental or nuclear specific factors have been suggested. Interestingly, two members of the family here (III.2 and IV.2) were found to be heterozygous for the 35delG mutation in the connexin 26 ( $\mathrm{C} \times 26)$ gene, the most common genetic cause of childhood hearing impairment. ${ }^{10,11}$ Individual IV.1 was homozygous for the wild type. The presence of this mutation had no apparent effect on the onset of severity of hearing loss in those with the A7445G mutation.

The finding of a fourth, independent, family with hearing impairment and the A7445G mutation implies it may be more frequent than previously thought. Other surveys suggest the A1555G and A3243G mutations are the most common cause of maternally inherited NSSHI in some populations. ${ }^{12-14}$ Although the A7445G mutation was not found in these surveys, nor in nine other families with possible matrilineal NSSHI we studied, our study shows the importance of screening for this mutation in cases of NSSHI and also highlights the difficulty sometimes encountered in establishing mitochondrial inheritance.

\section{Acknowledgements}

Work in the author's laboratories is supported by the National Lottery Charities Board through D efeating Deafness, the West Riding M edical Research Trust, the Medical Research Council, European Community, Wellcome Trust and Yorkshire Cancer Research.

We are grateful to the family for their co-operation.

\section{References}

1 Prezant TR, Agapian JV, BohIman MC et al: Mitochondrial ribosomal RNA mutation associated with both antibiotic-induced and non-syndromic deafness. Nat Genet 1993; 4: 289-294.

2 Hutchin TP: Sensorineural hearing loss and the $1555 \mathrm{G}$ mitochondrial DNA mutation. Acta Otolaryngol (Stockh) 1999; 119: 48-52.

3 Reid FM, Vernham GA, Jacobs HT: A novel mitochondrial point mutation in a maternal pedigree with sensorineural deafness. Hum Mutat 1994; 3: 243-247.

4 Hutchin TP, Parker MJ, Young ID et al: A novel mutation in the mitochondrial tRNA ${ }^{\operatorname{Se}(U C N)}$ gene in a family with maternally inherited hearing impairment. J Med Genet 2000; 37: 692-694.

5 Sue CM, Tanji K, Hadjigeorgiou GM et al: Maternally inherited isolated hearing loss in a large kindred with a novel T7511C mutation in the mitochondrial DNA tRNA(Ser(UCN)) gene. Neurology 1999; 52: 1905-1908.

6 Verhoeven K, Ensink RJH, Tiranti V et al: Hearing impairment and neurological dysfunction associated with a mutation in the mitochondrial tRNASer(UCN) gene. Eur J Hum Genet 1999; 7: 45-51.

7 Fischel-Ghodsian N, Prezant TR, Fournier P, Stewart IA, Maw M: Mitochondrial tRNA mutation associated with nonsyndromic deafness. Am J Otolaryngol 1995; 16: 403-408.

8 Sevior KB, Hatamochi A, Stewart IA et al: Mitochondrial A7445G mutation in two pedigrees with palmoplantar keratoderma and deafness. Am J Med Genet 1998; 75: 179-185.

9 Reid FM, Vernham GA, Jacobs HT: Complete mtDNA sequence of a patient in a maternal pedigree with sensorineural deafness. Hum Mol Genet 1994; 3: 1435-1436.

10 Denoyelle F, Weil D, Maw MA et al: Prelingual deafness: high prevalence of a 30 delG mutation in the connexin 26 gene. Hum Mol Genet 1997; 6: 2173-2177.

11 Zelante L, Gasparini P, Estivill X et al: Connexin 26 mutations associated with the most common form of non-syndromic autosomal recessive deafness (DFNB1) in Mediterraneans. Hum Mol Genet 1997; 6: 1605-1609.

12 Estivill X, Govea N, Barcelo E et al: Familial progressive sensorineural deafness is mainly due to the mtDNA A1555G mutation and is enhanced by treatment of aminoglycosides. Am J Hum Genet 1998; 62: 27-35.

13 Usami S, Abe S, Akita J et al: Prevalence of mitochondrial gene mutations among hearing impaired patients. J Med Genet 2000; 37: 38-40.

14 Lehtonen MS, Uimonen S, Hassinen IE, Majamaa K: Frequency of mitochondrial DNA point mutations among patients with familial sensorineural hearing impairment. Eur J Hum Genet 2000; 8: 315-318. 